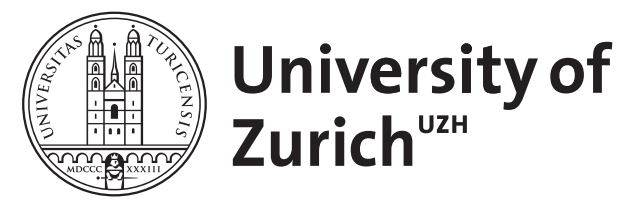

Zurich Open Repository and Archive

University of Zurich

University Library

Strickhofstrasse 39

CH-8057 Zurich

www.zora.uzh.ch

Year: 2005

\title{
About the explicit characterization of Hamiltonians of the Camassa-Holm hierarchy
}

Loubet, E

Abstract: We present a detailed computation leading to an explicit formula for the fourth Hamiltonian in the series of constants of motion with which any flow of the Camassa-Holm hierarchy is equipped, and explain the inherent difficulties in achieving such explicit expressions for invariants higher in the series.

DOI: https://doi.org/10.2991/jnmp.2005.12.1.11

Posted at the Zurich Open Repository and Archive, University of Zurich

ZORA URL: https://doi.org/10.5167/uzh-21734

Journal Article

Originally published at:

Loubet, E (2005). About the explicit characterization of Hamiltonians of the Camassa-Holm hierarchy. Journal of Nonlinear Mathematical Physics, 12(1):135-143.

DOI: https://doi.org/10.2991/jnmp.2005.12.1.11 


\title{
About the Explicit Characterization of Hamiltonians of the Camassa-Holm Hierarchy
}

\section{Enrique LOUBET}

Institut für Mathematik, Universität Zürich Winterthurerstrasse 190, CH-8057 Zürich, Switzerland.

E-mail: eloubet@math.unizh.ch

Received July 28, 2004; Accepted September 22, 2004

\begin{abstract}
We present a detailed computation leading to an explicit formula for the fourth Hamiltonian in the series of constants of motion with which any flow of the Camassa-Holm hierarchy is equipped, and explain the inherent difficulties in achieving such explicit expressions for invariants higher in the series.
\end{abstract}

\section{Introduction}

The equation of Camassa and Holm $[2,3]$ is an approximate one-dimensional description of unidirectional propagation of long waves in shallow water. In dimensionless space-time $\mathbb{R} \times[0,+\infty) \ni(x, t)$ variables it reads

$$
\frac{\partial m}{\partial t}=(m D+D m)(-v),
$$

in which $D=\partial / \partial x$, the real valued function $v$ represents the fluid velocity (or equivalently the height of the water's free surface above flat bottom), and $m=v-v^{\prime \prime}$ : in extenso,

$$
\frac{\partial v}{\partial t}-\frac{\partial^{3} v}{\partial t \partial x^{2}}+3 v \frac{\partial v}{\partial x}-2 \frac{\partial v}{\partial x} \frac{\partial^{2} v}{\partial x^{2}}-v \frac{\partial^{3} v}{\partial x^{3}}=0 .
$$

Its Eulerian form is more attractive: In terms of Green's function ${ }^{1} \mathrm{G}=\left(1-D^{2}\right)^{-1}$, it reads

$$
\frac{\partial v}{\partial t}+v \frac{\partial v}{\partial x}+\frac{\partial p}{\partial x}=0 \quad \text { with the "pressure" } \quad p=\mathrm{G}\left[v^{2}+\frac{1}{2}\left(v^{\prime}\right)^{2}\right] .
$$

It is reminiscent of the three-dimensional incompressible equations

$$
\frac{\partial v}{\partial t}+(v \cdot \operatorname{grad}) v+\operatorname{grad} p=0,
$$

\section{Copyright (c) 2005 by E Loubet}

\footnotetext{
${ }^{1}$ In terms of the kernels: $G=\exp \{-|x-y|\} / 2$ for the case on the line, and $G=\operatorname{Ch}\left[\frac{1}{2}\left(\frac{1}{2}-\{\lceil x-y\rceil-(x-y)\}\right)\right] / \operatorname{Sh}\left(\frac{1}{4}\right)$ for the analogue on the circle.
} 
in which $\triangle p \equiv \operatorname{sp}(\partial v / \partial x)^{2}$ and $\mathrm{G}$ is inverse to $-\triangle$, i.e. $\mathrm{G}=(4 \pi|x-y|)^{-1}$, and $p=$ $\mathrm{G}\left[\operatorname{sp}(\partial v / \partial x)^{2}\right]$.

This model was first noticed by Fokas and Fuchssteiner [10], by the method of recursion operators, as a formally integrable bi-Hamiltonian generalization of $\mathrm{KdV}$, but it became the subject of serious study after it was revamped from physical principles by Camassa and Holm $[2]^{2}$. Unlike this well-known ancestor, which is produced by approximation at the leading edge, $\mathrm{CH}$ was found in the course of approximating directly in the Hamiltonian for Euler's equations in the shallow water regime $[2]^{3}$. It is a good approximation for the full inviscid water wave equation, just as consistent in the small amplitude, shallow water regime as $\mathrm{KdV}$. But more is true, the $\mathrm{CH}$ equation is remarkable, as compared to $\mathrm{KdV}$, (a) for its peaked solitons and the simplicity of their interactions, (b) for its equivalence to the geodesic flow in the group of compressible diffeomorphisms of the line or of the circle (i.e. the periodic case [18]), and (c) for the presence of breaking waves. The bulk of basic material pertaining to "CH and all that" can be traced back to Camassa and Holm $[2,3]$. Speaking further about (a), (b), and (c), we point out that Beals, Sattinger and Szmigielski [1] adapted beautiful formulae by Stieltjes [19] to solve CH in the "multisoliton" case where $m \mathrm{~d} x$ reduces to a finite collection of signed point masses that lead to explicit formulas for solition interactions. Their results can be regarded as an extreme case of McKean's treatment of the case on the line which involves theta-like Fredholm determinants [14]. A complete account of the finite gap periodic case can be found in Constantin and McKean [7]. It is interesting to note that the geometric interpretation that (1.2) affords, leads to a confirmation of the Least Action Principle [6, 12]. On the other hand, [4] established that the only way in which singularities may arise for a classical solution of (1.2) is in the form of breaking waves in the sense of Whitham [20], i.e. that while the profile stays bounded, its slope becomes unbounded in finite time. Criteria ensuring breakdown can be found in $[5,16,14,16]$. Moreover, it is interesting to stress that in the case where no solitons are present, $\mathrm{KdV}$ and $\mathrm{CH}$ share a deeper kindship than their respective derivations might have suggested [15]. In short, since it was revamped, $\mathrm{CH}$ has been the subject of extensive investigations and by now has secured its place in the list of remarkable infinite-dimensional integrable nonlinear equations. Here, we do not entertain with any of the rich phenomena connected with this model. Rather, we focus on the well-known fact that such an infinite-dimensional integrable system admits, implicitly, an infinite number of independent conserved functionals. Their explicit expressions are important for applications. Indeed, Constantin and Strauss [8] used the first three invariants to establish, via energy estimates, the orbital stability of the peaked solitons. On the other hand, it is well known that in the case of $\mathrm{KdV}$, the constants of motion control higher and higher Sobolev norms, implying smoothness of the profile. This can no longer be true for the CH equation since "peaks" are present, but how can this be suspected from the explicit transcriptions of its invariants ${ }^{4}$ ? We offer a direct explicit computation of the next invariant in the sequence that starts with the most primitive ones and explain the inherent difficulties in deriving explicitly subsequent constants of motion by the recursion

\footnotetext{
${ }^{2}$ Consult [11] for a derivation using consistent asymptotic expansions in the governing equations for water waves.

${ }^{3}$ More recently, (1.2) has risen as a model for nonlinear waves in cylindrical axially symmetric hyperbolic rods, with $v$ representing the radial stretch relative to a pre-stressed state [9].

${ }^{4}$ See remark 4.
} 
method. We close the discussion by signaling that, in practice, alternative descriptions of the invariants also fail in producing, in a systematic fashion, concrete expressions for the independent Hamiltoninans.

\section{Explicit Computation of the $4^{\text {th }} \mathrm{CH}-\mathrm{Hamiltonian}$}

Either expressed in terms of the velocity field $v$ or the "mass" potential $m=v-v^{\prime}$, the first CH-Hamiltonians are ${ }^{5}$

$$
\begin{aligned}
& H_{0}=\int v=\int m, \\
& H_{1}=\frac{1}{2} \int\left(v^{2}+v^{\prime 2}\right)=\frac{1}{2} \int m G[m], \\
& H_{2}=\frac{1}{2} \int v\left(v^{2}+v^{\prime 2}\right)=\frac{1}{2} \int G[m]\left(G[m]^{2}+\{G[m]\}^{\prime 2}\right) .
\end{aligned}
$$

More explicitly these invariants are functionals ${ }^{6}$

$$
H[m]=H \circ\left(1-D^{2}\right)[v],
$$

from which it is plain that functional gradients with respect to $m$, and $v$ respectively, are related to each other. Indeed, let $w \equiv\left(1-D^{2}\right)(p)$ denote a variation of the potential $m$, and $p$ the effect of the latter registered along $v$. Then ${ }^{7}$

$$
\left.\frac{d}{d \epsilon} H[m+\epsilon w]\right|_{\epsilon=0}=d_{m} H[w]=<w, \overline{\partial H / \partial m}>_{0},
$$

is nothing but

$$
\left.\frac{d}{d \epsilon}\left(H \circ\left(1-D^{2}\right)\right)[v+\epsilon p]\right|_{\epsilon=0}=d_{v}\left\{H \circ\left(1-D^{2}\right)\right\}[p]=<p, \overline{\partial\left\{H \circ\left(1-D^{2}\right)\right\} / \partial v}>_{0} .
$$

Since the variation is arbitrary, and the operator $\left(1-D^{2}\right)$ is self-adjoint with respect to $H^{0}$-inner product, we learn that $\left(1-D^{2}\right)(\partial H / \partial m)=\partial\left\{H \circ\left(1-D^{2}\right)\right\} / \partial v$.

Remark 1. This is could have been expected from the formal chain rule $\partial H / \partial m=$ $\partial H / \partial\left(v-v^{\prime \prime}\right)=\left(1-D^{2}\right)^{-1}(\partial H / \partial v)$.

Warning 1. In the sequel we will use the symbols $h \equiv \partial H / \partial m$, and ${ }^{8} h^{\sharp} \equiv \partial H / \partial v$ respectively to denote the $H^{0}$-functional gradients. Hence the last result reads

$$
h^{\sharp}=\left(1-D^{2}\right)(h)=h-h^{\prime \prime} .
$$

\footnotetext{
${ }^{5}$ The present considerations hold for either the periodic case or the case on the line (with suitable decay at infinity of $v$ ). Therein the reason why we keep the domain of integration deliberately unspecified. More significantly, in either of these two cases, boundary terms arising when integrating by parts wash out.

${ }^{6}$ The underlying function spaces where $v$ and thus $m$ live, are such that the expressions for the invariants are well-defined, and for which associated $H^{0}$-gradients make sense.

${ }^{7}<\cdot, \cdot>_{0}$ denotes the standard $H^{0}$-scalar product.

${ }^{8} \partial H / \partial v$ is of course short for $\partial\left\{H \circ\left(1-D^{2}\right)\right\} / \partial v$.
} 
Now, it is a standard fact that the number of invariants under any flow of the CHhierarchy is infinite [3]. This is a byproduct of the bi-Hamiltonian structures in terms of which such flows may be expressed, i.e. the Lénard raising/lowering rule [13] by which corresponding Hamiltonian vector fields are identified. More precisely, we have the

\section{Poisson Structures.}

$$
\begin{aligned}
\mathcal{J} & \equiv m D+D m, \\
\mathcal{K} & \equiv D\left(1-D^{2}\right),
\end{aligned}
$$

connecting the functional gradients of subsequent Hamiltonians in the (countably infinite) list via the

\section{Lénard rule.}

$$
\mathcal{J}\left(h_{n}\right)=\mathcal{K}\left(h_{n+1}\right), \quad \text { for } \quad n \geq 0 .
$$

In symbols: $H_{n} \uparrow H_{n+1} \downarrow H_{n}$; the up $\uparrow /$ down $\downarrow$ arrows expressing the fact that the Hamiltonian raises/lowers.

Warning 2. For clarity, we will stick to the gradients throughout the sequel with the understanding that they arise from computations involving an integral in the background.

These are all the items we need for the actual computation of $H_{3}$. Indeed, by integration by parts

$$
\begin{aligned}
h_{2}^{\sharp} & =\left(3 v^{2}+v^{\prime 2}-2\left(v v^{\prime}\right)^{\prime}\right) / 2 \\
& =\frac{3}{2} v^{2}+\frac{1}{2} v^{\prime 2}-\left(\frac{1}{2} v^{2}\right)^{\prime \prime} .
\end{aligned}
$$

We will actually need $h_{2}$ rather than $h_{2}^{\sharp}$. Now $(2.2)$, dictates that $h_{2}=G\left[h_{2}^{\sharp}\right]$, hence all we need to do is to factor out the operator $\left(1-D^{2}\right)$ from the above display. Trivially,

$$
h_{2}^{\sharp}=\left(1-D^{2}\right)\left(\frac{1}{2} v^{2}\right)+v^{2}+\frac{1}{2} v^{2},
$$

so that, up to possible element in the kernel of $\left(1-D^{2}\right)$ that we ignore, the functional gradient $h_{2}$ reads

$$
h_{2}=\frac{1}{2} v^{2}+G\left[v^{2}+\frac{1}{2} v^{\prime 2}\right] \text {. }
$$

Remark 2. Notice that the above result is consistent with the original recipe ${ }^{9}+$ $\mathcal{J}\left(h_{1}\right)=0$ defining $\mathrm{CH}$ equation (1.1). Indeed, modulo the "factor" $\left(1-D^{2}\right)$, and the identification $\mathcal{J}\left(h_{1}\right)=\mathcal{K}\left(h_{2}\right)$, the latter reads $v^{\bullet}+\left(h_{2}\right)^{\prime}=0$, i.e. (1.2) as (2.4) would indicate.

\footnotetext{
${ }^{9}(\cdot)^{\bullet}$ is short for $\partial(\cdot) / \partial t$.
} 
On the other hand, the Lénard rule in combination with (2.2) yield $\mathcal{J}\left(h_{2}\right)=\mathcal{K}\left(h_{3}\right)=$ $\left(h_{3}^{\sharp}\right)^{\prime}$, which prompts writing $\mathcal{J}\left(h_{2}\right)=\left(m h_{2}\right)^{\prime}+m h_{2}^{\prime}$ as a total derivative. To this end, observe that

$$
\begin{aligned}
m h_{2}^{\prime} & =\left(v-v^{\prime \prime}\right) h_{2}^{\prime} \\
& =\left(v h_{2}-v^{\prime} h_{2}^{\prime}\right)^{\prime}-v^{\prime}\left(h_{2}-h_{2}^{\prime \prime}\right) \\
& =\left(v h_{2}-v^{\prime} h_{2}^{\prime}\right)^{\prime}-v^{\prime} h_{2}^{\sharp} .
\end{aligned}
$$

Now in order to spell the remaining item in the last display as a derivative, we invoke (2.3) and combine terms suitably as in

$$
\begin{aligned}
v^{\prime} h_{2}^{\sharp} & =v^{\prime}\left(\frac{3}{2} v^{2}-\frac{1}{2} v^{\prime 2}-v v^{\prime \prime}\right) \\
& =\left(\frac{1}{2} v^{3}\right)^{\prime}-\frac{1}{2} v^{\prime 3}-v v^{\prime} v^{\prime \prime} \\
& =\left(\frac{1}{2} v^{3}-\frac{1}{2} v v^{\prime 2}\right)^{\prime} .
\end{aligned}
$$

In other words, $m h_{2}^{\prime}=\left(v h_{2}-v^{\prime} h_{2}^{\prime}-v^{3} / 2+v v^{\prime 2} / 2\right)^{\prime}$, so that putting everything together

$$
\left(h_{3}^{\sharp}\right)^{\prime}=\left((m+v) h_{2}-v^{\prime} h_{2}^{\prime}-\frac{1}{2} v^{3}+\frac{1}{2} v v^{\prime 2}\right)^{\prime},
$$

i.e. $^{10}$

$$
\begin{aligned}
h_{3}^{\sharp} & =\left(2 v-v^{\prime \prime}\right) h_{2}-v^{\prime} h_{2}^{\prime}-\frac{1}{2} v^{3}+\frac{1}{2} v v^{\prime 2}+a \\
& =2 v h_{2}-\left(v^{\prime} h_{2}\right)^{\prime}-\frac{1}{2} v^{3}+\frac{1}{2} v v^{\prime 2}+a .
\end{aligned}
$$

The next step consists in identifying the right-hand-side of (2.5) as the functional gradient it should correspond to.

Remark 3. Here it is important to stress that the main difficulty lies in coping with terms which involve derivatives as these arise from terms (in the integrand defining the Hamiltonian) of either the form $v^{\alpha}\left\{D^{\gamma}(v)\right\}^{\beta}$ or $\left\{D^{\gamma}(v)\right\}^{\beta}$. Indeed, they originate from the latter from an intrinsic integration by parts which produces factors involving products of suitable derivatives, whereas, in conformity to Leibniz rule, they are generated from the former also via the functional differentiation of the factor $v^{\alpha}$. It is the assembling of the terms thus produced which makes the recovery of the Hamiltonian difficult when starting from its associated gradient.

Keeping the above remark in mind, we first observe that ${ }^{11} 2 v h_{2}-\left(v^{\prime} h_{2}\right)^{\prime}=h_{2} \partial\left(v^{2}+\right.$ $\left.1 / 2 v^{\prime 2}\right) / \partial v$, so that by Leibniz rule,

$$
2 v h_{2}-\left(v^{\prime} h_{2}\right)^{\prime}=\frac{\partial}{\partial v}\left\{\left(v^{2}+\frac{1}{2} v^{\prime 2}\right) h_{2}\right\}-\left(v^{2}+\frac{1}{2} v^{\prime 2}\right) \frac{\partial h_{2}}{\partial v} .
$$

\footnotetext{
${ }^{10} a$ denotes a constant.

${ }^{11} \mathrm{cf}$. warning 2 .
} 
But then switching momentarily to the symbolic shortcut $\mathfrak{q} \equiv\left(v^{2}+v^{\prime 2} / 2\right)$, a more convenient expression for $h_{3}^{\sharp}$ arises:

$$
h_{3}^{\sharp}=\frac{\partial\left(\mathfrak{q} h_{2}\right)}{\partial v}-\mathfrak{q} \frac{\partial h_{2}}{\partial v}-\frac{1}{2} v^{3}+v v^{\prime 2}+a .
$$

Indeed, recall (2.4) to express the last chunk as in ${ }^{12}$

$$
\begin{aligned}
-\mathfrak{q} \frac{\partial h_{2}}{\partial v}-\frac{1}{2} v^{3}+\frac{1}{2} v v^{\prime 2} & =-\mathfrak{q}\left(v+G\left[\frac{\partial \mathfrak{q}}{\partial v}\right]\right)-\frac{1}{2} v^{3}+\frac{1}{2} v v^{\prime 2} \\
& =-\frac{1}{2} \frac{\partial}{\partial v}(\mathfrak{q} G[\mathfrak{q}])-\frac{3}{2} v^{3} \\
& =-\frac{\partial}{\partial v}\left(\frac{1}{2} \mathfrak{q} G[\mathfrak{q}]+\frac{3}{8} v^{4}\right),
\end{aligned}
$$

so that when substituted back in $(2.6)$, the latter reads ${ }^{13}$

$$
\begin{aligned}
h_{3}^{\sharp} & =\frac{\partial}{\partial v}\left(\mathfrak{q} h_{2}-\frac{1}{2} \mathfrak{q} G[\mathfrak{q}]-\frac{3}{8} v^{4}\right)+a \\
& =\frac{\partial}{\partial v}\left(\frac{1}{2} v^{2} \mathfrak{q}+\frac{1}{2} \mathfrak{q} G[\mathfrak{q}]-\frac{3}{8} v^{4}+a v+b\right),
\end{aligned}
$$

in which $a$, and $b$ denote constants. Thence we can finish the job by simply infering what the third invariant with respect to $\mathrm{CH}$-flow has to be. Setting $a=b=0$ for simplicity $^{14}$, we conclude that

$$
H_{3}=\frac{1}{2} \int\left\{\left(v^{2}+\frac{1}{2} v^{\prime 2}\right) G\left[v^{2}+\frac{1}{2} v^{\prime 2}\right]+\frac{1}{4} v^{2}\left(v^{2}+2 v^{\prime 2}\right)\right\} .
$$

Remark 4. Notice that the expression within braces is non-local with respect to $v$. This is in sharp contrast to the $\mathrm{KdV}$ case, in which all integrands of invariant functionals correspond to homogeneous differential polynomials of the velocity potential $V^{15}$. More important is the fact that in this case only $v$ and $v^{\prime}$ intervene. Indeed, this discovery is in agreement to the fact that in the case of $\mathrm{CH}$ one cannot expect to control higher Sobolev norms of the solution because of the inherent possibility of breakdown.

Finally, we point out that if we mimic previous steps in order to figure out the next Hamiltonian in the series, we face an obstacle. The first couple of steps carry through without extra complications. Indeed, $\mathcal{J}\left(h_{3}\right)=\left(h_{4}^{\sharp}\right)^{\prime}$ where $\mathcal{J}\left(h_{3}\right)=\left(m h_{3}\right)^{\prime}+m h_{3}^{\prime}$. By

\footnotetext{
${ }^{12}$ Symmetry of the kernel $G$, the fact that $\mathfrak{q} v=v\left(v^{2}+v^{\prime 2} / 2\right)$, and Leibniz rule are used in passing to the second line below.

${ }^{13}$ As before we invoke (2.4) to come up with a final expression involving only $v$ and $\mathfrak{q}$.

${ }^{14}$ Notice that $H_{0}=\int v$, so that $a=0$ simply expresses the fact that $H_{3}$ is functionally independent of $H_{0}$, while $b=0$ enforces $H[0]=0$, i.e. that the density of no invariant contains constant terms.

${ }^{15} V$ stands for the velocity profile satistying the $\mathrm{KdV}$ equation: $\partial V / \partial t+V \partial V / \partial X+\partial^{3} V / \partial X^{3}=0$.
} 
direct computation

$$
\begin{aligned}
m h_{3}^{\prime} & =\left(v h_{3}-v^{\prime} h_{3}^{\prime}\right)^{\prime}-v^{\prime} h_{3}^{\sharp}, \\
v^{\prime} h_{3}^{\sharp} & =2 v v^{\prime} h_{2}-v^{\prime}\left(v^{\prime} h_{2}\right)^{\prime}-\frac{1}{2} v^{3} v^{\prime}+\frac{1}{2} v v^{\prime 3} \text {. Now } \\
2 v v^{\prime} h_{2}-v^{\prime}\left(v^{\prime} h_{2}\right)^{\prime} & =\left[\left(v^{2}-\frac{1}{2} v^{\prime 2}\right) h_{2}\right]^{\prime}-h_{2}^{\prime} \mathfrak{q} \quad \text { by integration by parts, } \\
\text { and } \quad h_{2}^{\prime} \mathfrak{q} & =\left(\frac{1}{4} v^{4}+\frac{1}{2} \mathfrak{q} G[\mathfrak{q}]\right)^{\prime}+\frac{1}{2} v v^{\prime 3} \text {. Hence } \\
v^{\prime} h_{3}^{\sharp} & =\left(\left(v^{2}-\frac{1}{2} v^{\prime 2}\right) h_{2}-\frac{1}{2} \mathfrak{q} G[\mathfrak{q}]-\frac{3}{8} v^{4}\right)^{\prime},
\end{aligned}
$$

so that upon reassembling all these pieces

$$
\left(h_{4}^{\sharp}\right)^{\prime}=\left((m+v) h_{3}-v^{\prime} h_{3}^{\prime}-\left(v^{2}-\frac{1}{2} v^{\prime 2}\right) h_{2}+\frac{1}{2} \mathfrak{q} G[\mathfrak{q}]+\frac{3}{8} v^{4}\right)^{\prime} .
$$

We infer, exactly as before, that

$$
h_{4}^{\sharp}=2 v h_{3}-\left(v^{\prime} h_{3}\right)^{\prime}-\left(v^{2}-\frac{1}{2} v^{\prime 2}\right) h_{2}+\frac{1}{2} \mathfrak{q} G[\mathfrak{q}]+\frac{3}{8} v^{4} .
$$

Comparing the latter with (2.5), suggests going one step further by writing the first two terms above as in $h_{3} \partial \mathfrak{q} / \partial v=\partial\left(\mathfrak{q} h_{3}\right) / \partial v-\mathfrak{q} \partial h_{3} / \partial v$, so that

$$
\begin{aligned}
h_{4}^{\sharp} & =\frac{\partial}{\partial v}\left(\mathfrak{q} h_{3}+\frac{3}{40} v^{5}\right)+\mathcal{R} \\
\mathcal{R} & =-\mathfrak{q} \frac{\partial h_{3}}{\partial v}-\left(v^{2}-\frac{1}{2} v^{\prime 2}\right) h_{2}+\frac{1}{2} \mathfrak{q} G[\mathfrak{q}] .
\end{aligned}
$$

The problem now is that $\mathcal{R}$ does not offer any indication as to how to recast it as the functional gradient it should correspond to. In fact, we cannot even produce a "nice" expression for $h_{3}$ starting from (2.5) which we could then exploit with the hope of getting a more manageable expression, by which we mean, one from which terms containing deriviatives would offer some insight as to how to assimilate them as the result of computing a suitable gradient ${ }^{16}$. In short, the inherent difficulty in establishing the latter identification of (necessarily more and more complex) derivative terms, reveals the limited character of the recursive approach for producing explicitly the invariants.

Remark 5. An analogous iterative method for computing $\mathrm{KdV}$ invariants might give rise to similar obstructions. Fortunately, there is a way out in this case. Indeed, we can guess which combinations of $V, V^{\prime}, V^{\prime \prime} \ldots$ will show up in the integrands defining the $\mathrm{KdV}$ functionals since they correspond to homogenous (in some appropiate sense) differential polynomials in $V$, i.e. the "hands-on" computation of further KdV constants of motion boils down to the determination of the constant weights, multiplying each of the factors which conform to the homogeneity constraint. Actually, "homogeneity" of the integrands is not a problem in the case of $\mathrm{CH}$ as the first couple of invariants show: indeed,

\footnotetext{
${ }^{16}$ cf. remark 3 .
} 
upon declaring $\operatorname{deg}(v)=\operatorname{deg}\left(v^{\prime}\right) \equiv 1$, it follows that ${ }^{17} \operatorname{deg}[(2.1 a)]=1, \operatorname{deg}[(2.1 b)]=2$, $\operatorname{deg}[(2.1 c)]=3, \operatorname{deg}[(2.7)]=4$, etc.... Here the obstruction to "guess," i.e. to compute the constants factors, is rather due to the non-local character of the invariants.

To conclude we point out to the reader that, in the periodic case [7], there is an alternative way ${ }^{18}$ of potentially computing the invariants. It suggests itself to be more direct than the previous attempt in that it avoids passing through the gradients. Indeed, consider $\mathrm{Ch}^{-1}[\triangle(\lambda)]$ where $\triangle(\lambda)=\frac{1}{2} \times$ trace of the monodromy matrix, alias the discriminant which in turn is constructed from a pair of so called standard solutions of the acoustic problem $^{19}$. The latter invariant may be regarded as a generating function for all other ones since the McLaurin series of the former with respect to $2 \lambda$, embody as coefficients the aforementioned series of Hamiltonians ${ }^{20}$. To wit ${ }^{21}$,

$$
\begin{aligned}
-2 \mathrm{Ch}^{-1}[\triangle(\lambda)] & =-\left.2 \mathrm{Ch}^{-1}[\triangle]\right|_{0}-\left.\left(\frac{\triangle^{\bullet}}{\sqrt{\triangle^{2}-1}}\right)\right|_{0} \times(2 \lambda) \\
& -\left.\frac{1}{8}\left[\frac{-\triangle(\triangle \bullet)^{2}}{\left(\triangle^{2}-1\right)^{3 / 2}}+\frac{\triangle^{\bullet \bullet}}{\sqrt{\triangle^{2}-1}}\right]\right|_{0} \times(2 \lambda)^{2}+O\left((2 \lambda)^{3}\right) .
\end{aligned}
$$

Indeed, the constant term on the expansion can be computed immediately from the monodromy properties of standard solutions as Floquet theory would indicate. It corresponds to the period. For the computations pertaining to the remaining coefficients, we also need to make use of the integral expressions that the standard solutions satisfy to compute the values of the derivatives (at the origin) of the discriminant which arise in the expansion. Following this procedure, we recover as expected $\left.\triangle^{\bullet}\left(\triangle^{2}-1\right)^{-1 / 2}\right|_{0}=H_{0}$, and after some effort $H_{1}$, i.e. the coefficients associated to $(2 \lambda)$, and $(2 \lambda)^{2}$ respectively. We did not even pursue the verification that the next coefficient corresponds to $\mathrm{H}_{2}$ because the computations are lengthy, and more significantly, because they give indication of a sort of obstruction (analogous to that of the former method) that requires some extra thinking to breakthrough. In short, there does not seem to be an infallible systematic way for computing explicitly the invariants.

\section{Acknowledgments}

The author wishes to express his gratitude to the referee for several references and appropiate comments. Research partially supported by the Swiss National Science Foundation. This is gratefully acknowledged.

\section{References}

[1] Beals, R.; Sattinger, D. H.; Szmigielski, J., "Multipeakons and the classical moment problem" Adv. Math. 154 (2000), no. 2, 229-257.

\footnotetext{
${ }^{17} \mathrm{deg}$ (invariant) is short of course of the degree associated to the integrand of the latter.

${ }^{18} \mathrm{cf}$. [15] for details.

${ }^{19}[13]$ contains all necessary background.

${ }^{20}$ The Lénard rule follows from the identity $[\mathcal{K}-2 \lambda \mathcal{J}](\partial \mathrm{Ch}[\triangle] / \partial m)=0$.

${ }^{21}$ Below $(\cdot)^{\bullet}$ stands for differentiation with respect to $\lambda$.
} 
[2] Camassa, R.; Holm, D. D. "An integrable shallow water equation with peaked solitons" Phys. Rev. Lett. 71 (1993), no. 11, 1661-1664.

[3] Camassa, R.; Holm, D. D.; Hyman, M. "A new integrable shallow water equation" Adv. Appl. Math. 31 (1994), 1-33.

[4] Constantin, A. "Existence of permanent and breaking waves for a shallow water equation: a geometric approach" Ann. Inst. Fourier (Grenoble) 50 (2000), no. 2, 321-362.

[5] Constantin, A.; Escher, J. "Wave breaking for nonlinear nonlocal shallow water equations" Acta Mathematica. 181 (1998), no. 2, 229-243.

[6] Constantin, A.; Kolev, B. "On the geometric approach to the motion of inertial mechanical systems" J.Phys. A 35 (2002), no. 32, R51-R79.

[7] Constantin, A.; McKean, H.P. "A shallow water equation on the circle" Comm. Pure Appl. Math. 52 (1999), no. 8, 949-982.

[8] Constantin, A.; Strauss W. "Stability of peakons" Comm. Pure Appl. Math. 53 (2000), 603-610.

[9] DAI, H. "Model equations for nonlinear dispersive waves in a compressible Mooney-Rivlin rod" Acta Mech. 127 (1998), 193-207.

[10] Fokas,A.; Fuchssteiner,B. "Symplectic structures, their Bläcklund transformations and hereditary symmetries" Physica 4-D. (1981), 47-66.

[11] Johnson, R.S. "Camassa-Holm, Korteweg-de Vries, and related models for water waves" Journal of Fluid Mechanics 455 (2000), 63-82.

[12] Kolev, B. "Geodesic flow on the diffeomorphism group of the circle" Comment. Math. Helv. 78 (2003), no. 4, 787-804.

[13] McKean, H. P. "Compatible brackets in Hamiltonian dynamics" Springer Series in nonlinear Dynamics, A.Fokas, V.E. Zakharov (Eds) Important developments in Soliton Theory..

[14] McKean, H. P. "Fredholm determinants and the Camassa-Holm hierarchy" Comm. Pure Appl. Math. 56 (2003), no.5, 638-680.

[15] McKean, H. P. "The Liouville correspondence between the Korteweg-de Vries and the Camassa-Holm hierarchies" Comm. Pure Appl. Math. 56 (2003), no. 7, 0998-1015.

[16] McKean, H. P. "Breakdown of a shallow water equation" Asian J. Math. 2 (1998), no. 4, 867-874; corrected in Asian J. Math. 3 (1999), 3.

[17] McKean, H. P. "Breakdown of the Camassa-Holm equation" Comm. Pure Appl. Math. 57 (2004), 416-418.

[18] Misiolek,G. "A shallow water equation as a geodesic flow on the Bott-Visasoro group" $J$. Geom. Phys. 24 (1998), 203-208.

[19] Stieltues, T. J. "Recherches sur les fractions continues" Oeuvres complétes 2, 402-566. Nordhoff, Gröningen, 1918.

[20] Whitham, G.B. "Linear and Nonlinear Waves" John Wiley \& Sons, Inc., New York, 1999. 